\title{
Studying the properties of the radio emitter in LS 5039 (Research Note)
}

\begin{abstract}
V. Bosch-Ramon
Max Planck Institut für Kernphysik, Saupfercheckweg 1, Heidelberg 69117, Germany

e-mail: vbosch@mpi-hd.mpg.de

Received 16 September 2008 / Accepted 4 November 2008

ABSTRACT

Context. LS 5039 is an X-ray binary that presents non-thermal radio emission. The radiation at $\sim 5 \mathrm{GHz}$ is quite steady and optically thin, consisting of a dominant core plus an extended jet-like structure. There is a spectral turnover around $1 \mathrm{GHz}$, and evidence of variability on timescales of $1 \mathrm{yr}$ at $234 \mathrm{MHz}$.

Aims. We investigate the radio emitter properties using the available broadband radio data, and assuming two possible scenarios to explain the turnover: free-free absorption in the stellar wind, or synchrotron self-absorption.

Methods. We use the relationships between the turnover frequency, the stellar wind density, the emitter location, size and magnetic field, and the Lorentz factor of the emitting electrons, as well as a reasonable assumption regarding the energy budget, to infer the properties of the low-frequency radio emitter. Also, we put this information in context with the broadband radio data.

Results. The location and size of the low-frequency radio emitter can be restricted to $\gtrsim$ few AU from the primary star, its magnetic field to $\sim 3 \times 10^{-3}-1 \mathrm{G}$, and the electron Lorentz factors to $\sim 10-100$. The observed variability of the extended structures seen with VLBA would point to electron bulk velocities $\gtrsim 3 \times 10^{8} \mathrm{~cm} \mathrm{~s}^{-1}$, whereas much less variable radiation at $5 \mathrm{GHz}$ would indicate velocities for the VLBA core $\lesssim 10^{8} \mathrm{~cm} \mathrm{~s}^{-1}$. The emission at $234 \mathrm{MHz}$ in the high state would mostly come from a region larger than the dominant broadband radio emitter.

Conclusions. We suggest a scenario in which secondary pairs, created via gamma-ray absorption and moving in the stellar wind, are behind the steady broadband radio core, whereas the resolved jet-like radio emission would come from a collimated, faster, outflow.
\end{abstract}

Key words. radio continuum: stars - X-rays: binaries - stars: individual: LS 5039 - radiation mechanisms: non-thermal

\section{Introduction}

LS 5039 is an X-ray binary that presents broadband non-thermal emission: in radio (e.g. Martí et al. 1998; M98 hereafter); in X-rays (e.g. Bosch-Ramon et al. 2007); at GeV energies (Paredes et al. 2000; PA0); and at TeV energies (Aharonian 2005, 2006), as well as extended $\sim 10-1000$ AU radio jets (e.g. PA0; Paredes et al. 2002; Ribó et al. 2008, R08 hereafter). The mass of the compact object is not well constrained (Casares et al. 2005; C05 hereafter), and it may harbor an accreting blackhole (e.g. Paredes et al. 2006) or a non-accreting pulsar (e.g. Martocchia et al. 2005; Dubus 2006). The source is at a distance of $2.5 \mathrm{kpc}(\mathrm{C} 05)$.

Low-frequency ( $\$ 1 \mathrm{GHz}$ ) radio emission has been detected by the Giant Metrewave Radio Telescope - GMRT - (Pandey et al. 2007; Godambe et al. 2008; P07 and G08 hereafter) in LS 5039. This radio emission is very likely of synchrotron origin, matching well with the non-thermal radio spectrum at higher frequencies (see Fig. 2 in G08). G08 found a spectral turnover at $v_{\max }=964 \pm 104 \mathrm{MHz}$ (with a flux $F_{\max } \approx 40 \mathrm{mJy}$ ), fluxes of $\approx 17.0 \pm 1.1$ and $34.2 \pm 2.8 \mathrm{mJy}$ at 234 and $614 \mathrm{MHz}$, respectively, and a spectral index $\alpha=0.75 \pm 0.11\left(F_{v} \propto v^{\alpha}\right)$ in this frequency range. These data were taken simultaneously $(\mathrm{MJD}=53788.78)$. Above the spectral turnover, at $1.28 \mathrm{GHz}$, the flux was $38.1 \pm 2.3 \mathrm{mJy}$, this data point being obtained 18 days later $(\mathrm{MJD}=53806.73)$ than those at lower frequencies. Above the spectral turnover, $\alpha$ was $\approx-0.43$, very similar to that found by M98. The P07 observations, taken in two different epochs (MJD = 53220 and 53 377) more than 1 yr apart from the G08 observations, found significantly different fluxes at $234 \mathrm{MHz}$ : $65.04 \pm 7.71$ and $74.56 \pm 5.63 \mathrm{mJy}$, which is evidence of variability. Otherwise, the fluxes of P07 at $614 \mathrm{MHz}$ were $28.55 \pm 3.56$ and $34.29 \pm 2.34 \mathrm{mJy}(\mathrm{MJD}=53220,53377)$, similar to the value of G08. The $\alpha$ values found by P07 were $\approx-0.81$ and -0.86 .

The spectrum found at low frequencies by G08, far more inverted than the one corresponding to a monoenergetic distribution of particles (i.e. $\alpha=1 / 3$ ), could not be explained by an electron population with a high minimum energy, the most likely origin of the turnover being either free-free absorption in the stellar wind or synchrotron self-absorption. Based on this, we investigate in this work the properties of the low-frequency radio emitter in LS 5039 adopting a plain framework in which the radio emission, at different narrow frequency bands, is produced in a homogeneous spherical emitter with magnetic field $B$, size $r$, and at a distance $R \gtrsim r$ from the star/binary system. We also derive physical constraints of the broadband radio emitter from data at higher frequencies. Finally, we suggest a scenario for the radio emission, and a more complex model is applied to test its feasibility.

\section{Studying the low-frequency radio emission from LS 5039}

In this section, we infer basic properties of the low-frequency radio emitter in LS 5039 accounting for the detection of a spectral 


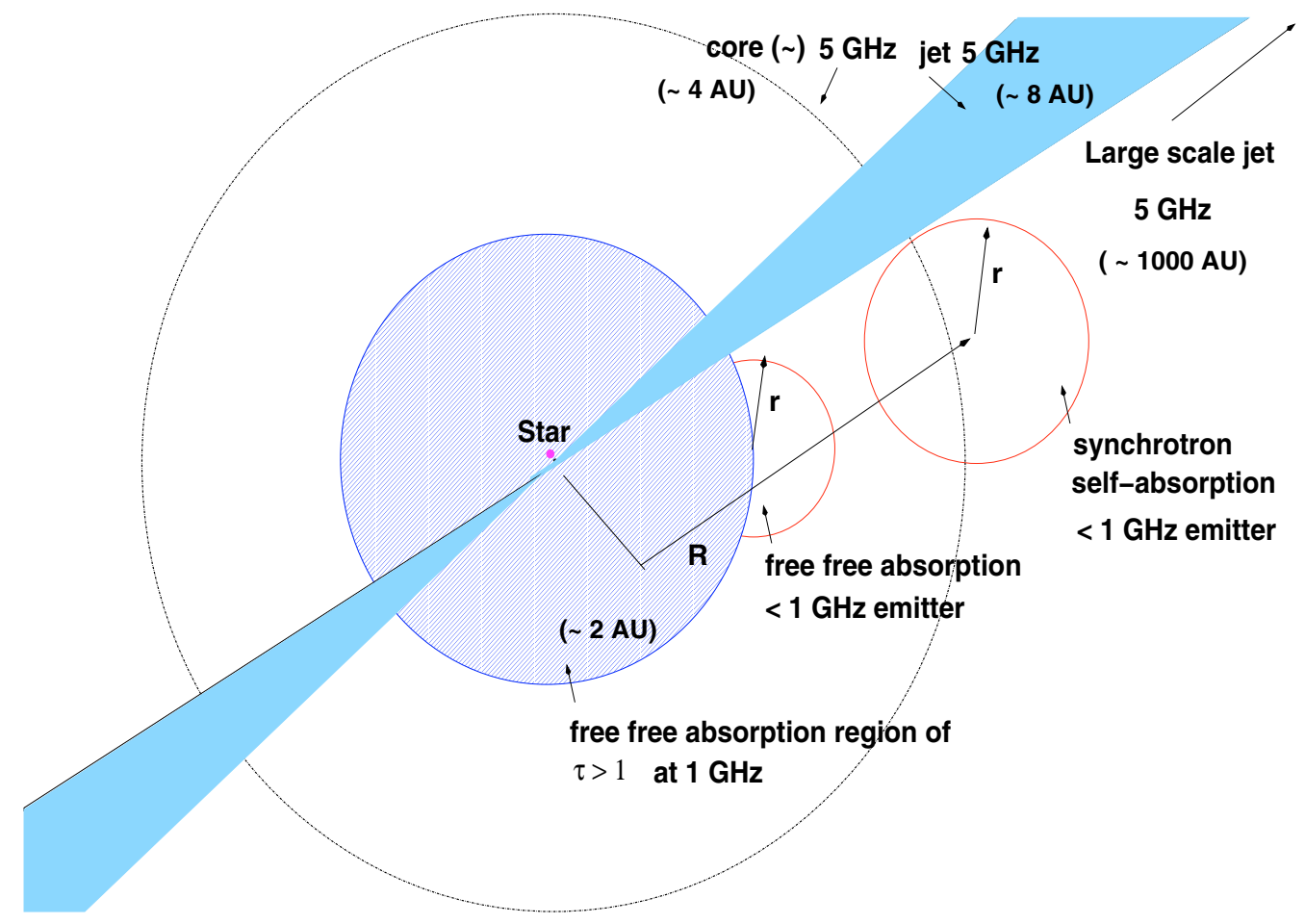

Fig. 1. Sketch of the regions producing radio emission in LS 5039. The main components of the radio emitter at low and high frequencies are shown, together with the relevant spatial scales.

turnover at $\approx 1 \mathrm{GHz}$ and the observational evidence of variability at $234 \mathrm{MHz}$.

For illustrative purposes, we present in Fig. 1 a sketch of the scenario considered here. In the picture, the low-frequency radio emitter location is illustrated for two cases: a low-frequency turnover produced by free-free absorption, with the emission coming from a region at a few AU from the star, or by synchrotron self-absorption, with the emitter located at similar or greater distances (see below). The wind region, optically thick by free-free absorption to $1 \mathrm{GHz}$ emission, is shown. The core and the jet-like emission at $5 \mathrm{GHz}$, discussed later, are also sketched.

\subsection{Free-free absorption}

The strongly inverted low-frequency radio spectrum could be produced by free-free absorption in the stellar wind. The distance at which the stellar wind becomes optically thin to radio emission can be computed fixing the free-free opacity $\tau_{v}$ ff (Rybicki \& Lightman 1979) to 1:

$\tau_{v \mathrm{ff}} \approx 30 \dot{M}_{-7}^{2} v_{\mathrm{GHz} \max }^{-2} R_{\mathrm{AU}}^{-3} V_{\mathrm{w} 8.3}^{-2} T_{\mathrm{w} 4}^{-3 / 2}=1$,

which, for $v_{\mathrm{GHz} \max }=v_{\max } / 1 \mathrm{GHz}=1$, yields:

$R \approx 3 \dot{M}_{-7}^{2 / 3} V_{\mathrm{w} 8.3}^{-2 / 3} T_{\mathrm{w} 4}^{-1 / 2} \mathrm{AU}$,

where $\dot{M}_{-7}=\dot{M} / 10^{-7} M_{\odot} \mathrm{yr}^{-1}$ is the stellar mass loss rate, $R_{\mathrm{AU}}=R / 1 \mathrm{AU}, V_{\mathrm{w}} 8.3=V_{\mathrm{w}} / 2 \times 10^{8} \mathrm{~cm} \mathrm{~s}^{-1}$ the stellar wind velocity, and $T_{\mathrm{w} 4}=T_{\mathrm{w}} / 10^{4} \mathrm{~K}$ the wind temperature. Adopting $T_{\text {w } 4}=1$ (Krtidka \& Kubát 2001), $V_{8.3}=1\left(\right.$ C05), and $\dot{M}_{-7}=1$, we obtain $R \approx 3 \mathrm{AU}\left(4.5 \times 10^{13} \mathrm{~cm}\right.$; recall that $\left.r \lesssim R\right)$ as the distance between the low-frequency radio emitter and the star.

A $\dot{M}$ somewhat lower than the values given by $\mathrm{C} 05$ has been taken to account for possible wind clumpiness in LS 5039
(Bosch-Ramon et al. 2007). Actually, wind clumpiness may prevent the wind from being an effective free-free absorber for large enough porosity lengths (e.g. Owocki \& Cohen 2006). In such a case, if a pulsar is present in the system, the radio pulse is not smeared out, hence being detectable. No radio pulsations have been found up to now.

If the spectral turnover is produced by wind free-free absorption, the synchrotron self-absorption frequency must be $<1 \mathrm{GHz}$. Thus, to find a restriction for $B$, one can use the following relationship between $v_{\max }, F_{\max }, r$ and $B$ in the synchrotron selfabsorption case:

$$
v_{\max } \approx 3.9 \times 10^{10} F_{\max m \mathrm{my}_{\mathrm{G}}^{2 / 5}}^{1 / 5} r_{\mathrm{cm}}^{-4 / 5} \mathrm{GHz}
$$

where $F_{\max } \mathrm{mJy}=F_{\max } / \mathrm{mJy}$, and $r_{\mathrm{cm}}=r / \mathrm{cm}$. Since $F_{\max \text { mJy }}=$ $F_{\max } / \mathrm{mJy}=40$ and $r \lesssim R \approx 4.5 \times 10^{13} \mathrm{~cm}$, the condition $v_{\max }<1 \mathrm{GHz}$ imposes that $B \lesssim 3 \times 10^{-2} \mathrm{G}$.

The constraint for $B$, together with the following formula (e.g. Pacholczyk 1970):

$v \approx 4.2 \times 10^{-3} B_{\mathrm{G}} \gamma^{2} \mathrm{GHz}$,

allows us to constrain the Lorentz factor of the electrons emitting at $1 \mathrm{GHz}$ to $\gamma \gtrsim 10^{2}$.

Further restrictions on $r, B$ and $\gamma$ can be derived from energy budget constraints. On the one hand, the synchrotron cooling timescale for $1 \mathrm{GHz}$ emitting electrons is:

$t_{\mathrm{sync}} \approx 5 \times 10^{8} B_{\mathrm{G}}^{-2} \gamma^{-1} \approx 3.3 \times 10^{7} B_{\mathrm{G}}^{-3 / 2} \mathrm{~s}$.

In general, radio electrons will be transported/advected out of the emitter well before radiating all their energy via synchrotron emission, i.e. $t_{\mathrm{adv}} \ll t_{\text {sync }}$. For reasonable advection velocities $v_{\text {adv }} \gtrsim 2 \times 10^{8} \mathrm{~cm} \mathrm{~s}^{-1}$ and emitter sizes $r \lesssim R \approx 4.5 \times 10^{13} \mathrm{~cm}$ (the free-free absorption case), one gets $t_{\mathrm{adv}} \lesssim 2 \times 10^{5} \mathrm{~s}$, where 
$v_{\text {adv }}$ has been taken assuming that the emitter flow cannot move slower than the stellar wind. On the other hand, the total broadband non-thermal luminosity of the source, $L_{\mathrm{e}} \sim 10^{36} \mathrm{erg} \mathrm{s}^{-1}$, can be taken as an upper limit for the injected luminosity in the form of radio emitting electrons. Finally, the observed lowfrequency luminosity is $L_{\mathrm{GHz}} \sim 10^{30} \mathrm{erg} \mathrm{s}^{-1}$. From all this, we can write:

$t_{\text {sync }} \lesssim t_{\text {adv }}\left(L_{\mathrm{e}} / L_{\mathrm{GHz}}\right) \lesssim 2 \times 10^{11} \mathrm{~s}$.

Therefore, accounting for Eqs. (4)-(6), we derive $B \gtrsim 3 \times$ $10^{-3} \mathrm{G}$ and $\gamma \lesssim 3 \times 10^{2}$, which allow us to restrict $B$ to $\sim(3-30) \times 10^{-3} \mathrm{G}$, and $\gamma$ to $\sim(1-3) \times 10^{2}$. The minimum $B$-value, and $v_{\max }<1 \mathrm{GHz}$, and $r<R$, imply that $r \sim$ $(3-4.5) \times 10^{13} \mathrm{~cm}$.

\subsection{Synchrotron self-absorption}

If synchrotron self-absorption is the origin of the turnover, we can obtain, from Eqs. (3) and (4), $v_{\max }=1 \mathrm{GHz}$, and $F_{\max } \approx$ $40 \mathrm{mJy}$, the following $r-B$ and $r-\gamma$ relationships:

$r \approx 10^{14} B_{\mathrm{G}}^{1 / 4} \approx 4 \times 10^{14} \gamma^{-1 / 2} \mathrm{~cm}$.

Since the synchrotron emitting electrons must be relativistic, $\gamma \gtrsim$ 10 , and therefore, $r \lesssim 10^{14} \mathrm{~cm}$ and $B \lesssim 1 \mathrm{G}$.

In this case, having a constraint on $r$, the argument of the limited energy budget can be applied as well, allowing us to derive further restrictions on the parameters: $B \sim 10^{-2}-1 \mathrm{G}$; $\gamma \sim 10-10^{2}$; and $r \sim(4.5-10) \times 10^{13} \mathrm{~cm} \sim$ few $\mathrm{AU}$ (recall $R \gtrsim r)$.

\subsection{Variable emission at $234 \mathrm{MHz}$}

The combination of P07 and G08 observations show variability at $234 \mathrm{MHz}$ of $400 \%$ on timescales of about one year, too large to be attributed only to interstellar medium scintillation effects. The flux at $614 \mathrm{MHz}$ does not show significant changes. The value of $t_{\mathrm{sync}}$ at $234 \mathrm{MHz}$ is:

$t_{\text {sync }}=1.4 \times 10^{8} B_{\mathrm{G}}^{-3 / 2} \mathrm{~s}$,

probably too long to explain the variability just with changes of the electron injection. Some level of variability around $1 \mathrm{GHz}$ may be induced by changes in the stellar wind if free-free absorption produced the spectral turnover, but at the estimated distance for the free-free absorption case, synchrotron selfabsorption should still affect the radiation at $234 \mathrm{MHz}$ due to the compactness of the region.

The optically thin nature of P07 data, and the fast variability, can otherwise be explained by advection with timescales shorter than $\sim 1$ yr together with injection variations. Taking $v_{\text {adv }} \lesssim 10^{10} \mathrm{~cm} \mathrm{~s}^{-1}$, the $\sim 1$ yr timescale restricts the size where the (high-state) $234 \mathrm{MHz}$ radiation is produced to $r \lesssim 3 \times$ $10^{17} \mathrm{~cm}$. $v_{\text {adv }}$ has been taken here to be at most mildly relativistic to avoid the complexities of significant boosting effects (on the other hand not expected; see Paredes et al. 2000, 2002). In addition, the fact that this emission is not synchrotron self-absorbed in the P07 data, and the flux at $234 \mathrm{MHz} \approx 70 \mathrm{mJy}$, implies a size:

$r \gtrsim 9 \times 10^{14} B_{\mathrm{G}}^{1 / 4} \approx 4 \times 10^{15} \gamma^{-1 / 2} \mathrm{~cm}$.

Accounting for energy budget constraints, the $1 \mathrm{yr}$ variability, and the constraint $\gamma \gtrsim 10$ (i.e. electrons must be relativistic), in Eq. (9), we get: $B \sim 4 \times 10^{-4}-0.6 \mathrm{G} ; \gamma \sim 10-400$; and $r \sim R \sim 10^{14}-3 \times 10^{17} \mathrm{~cm} . R$ has similar limits to those of $r$ if bigger, the source would have appeared as extended under the GMRT resolution of $\sim 10 \operatorname{arcsec}$ at $234 \mathrm{MHz}$.

Thus, the high-state $234 \mathrm{MHz}$ emitting region probably has a larger size and smaller magnetic field than the region producing the emission $\geq 614 \mathrm{MHz}$ and at $234 \mathrm{MHz}$ in the low-state, which indicates that they are probably different. The different spectral shape of P07 data and data $>614 \mathrm{MHz}$ would also point to a different electron population. Otherwise, despite the variability at $234 \mathrm{MHz}$, the quite steady $614 \mathrm{MHz}$ flux makes the conclusions of Sect. 2.1 still valid concerning the break at $\approx 1 \mathrm{GHz}$.

In Table 1, at the top, the results of this section are summarized.

\section{The broadband radio emission}

\subsection{The $5 \mathrm{GHz}$ VLBA radio emission}

The core seen with VLBA at $5 \mathrm{GHz}$ in LS 5039 is not point like, presenting a projected size of $\sim 8 \mathrm{AU}$, and two additional south-east (SE) and north-west (NW) components at a projected distance of $\sim 8 \mathrm{AU}$ from the core center are also detected (PA0; $\mathrm{R} 08$ ). The variation of the core flux at $5 \mathrm{GHz}$ within 5 days is about a $10 \%$, whereas the changes in flux of the SE and NW components are of about a 44 and a $260 \%$, respectively (R08). Assuming that the flux changes of the SE and NW component indicate power engine variations (unnecessarily affecting both components in the same way), plus the fact that the synchrotron cooling timescales of radio electrons are too long to explain such a variability, we can derive that $v_{\text {adv }} \lesssim 10^{8} \mathrm{~cm} \mathrm{~s}^{-1}$ to have a steady core, and $\gtrsim 3 \times 10^{8} \mathrm{~cm} \mathrm{~s}^{-1}$ for the variable SE and NW components. If shorter time variability in the SE and NW components were observed, it would increase the $v_{\text {adv }}$ lower limit to $3 \times 10^{8}(t / 5 \text { days })^{-1} \mathrm{~cm} \mathrm{~s}^{-1}$. The main properties of the VLBA radio emission are summarized in Table 1 , bottom.

Since LS 5039 harbors a very bright star (C05) and produces $\mathrm{TeV}$ emission (Aharonian et al. 2005), secondary pairs can be efficiently produced in the stellar wind via gamma-ray absorption. This population of secondary pairs could be behind the radio core (e.g. Bosch-Ramon et al. 2008a; B08 hereafter), moving collectively with the stellar wind, hence the low inferred advection speed. The feasibility of this scenario is illustrated in Fig. 2, where we show the computed radio spectral energy distribution (SED) of the secondary synchrotron emission created by gamma-ray absorption in LS 5039. The magnetic field on the stellar surface has been fixed to $250 \mathrm{G}$ (expected to be reasonable; see Bosch-Ramon et al. 2008b), the injected TeV luminosity to $4 \times 10^{35} \mathrm{erg} \mathrm{s}^{-1}$ (similar to that inferred by Khangulyan et al. 2008), and the TeV emitter distance to the stellar companion to $3.5 \times 10^{12} \mathrm{~cm}$, not exactly inside the binary system (as proposed in Bosch-Ramon et al. 2008b).

As seen in Fig. 2, the computed fluxes match the observed values, and most of the $5 \mathrm{GHz}$ emission is predicted to come from a region of a few AU in size, similar to first order to the values discussed in the previous paragraph. The computed $\alpha$ around $5 \mathrm{GHz}$ is $\approx-0.4$, roughly the same as the value found by M98 for the VLA radio emission (which should be dominated by the VLBA core). The computed low-frequency spectrum, similar to the observed one (G08), suggests that this radiation may come also from secondary pairs, although we note that synchrotron self-absorption has been roughly computed supposing a homogeneous emitter as wide as it is long. Also, free-free absorption in the stellar wind has not been account for. The magnetic field where most of the emission $<1 \mathrm{GHz}$ is produced, at a distance of several AU from the star, is $\sim 1 \mathrm{G}$, in agreement with the values 
Table 1. Basic physical parameters of the radio emitter in LS 5039.

\begin{tabular}{|c|c|c|c|c|c|c|}
\hline Case & \multicolumn{2}{|l|}{$\begin{array}{l}B \\
{[\mathrm{G}]}\end{array}$} & \multicolumn{2}{|l|}{$\gamma$} & $\begin{array}{l}r \\
{[\mathrm{~cm}]}\end{array}$ & $\begin{array}{l}R \\
{[\mathrm{~cm}]}\end{array}$ \\
\hline $\begin{array}{c}\text { free-free abs. } \\
\text { synchrotron self-abs. } \\
234 \mathrm{MHz} \text { variability }\end{array}$ & \multicolumn{2}{|c|}{$\begin{array}{l}\sim(3-30) \times 10^{-3} \\
\sim 10^{-2}-1 \\
\sim 4 \times 10^{-4}-0.6\end{array}$} & \multicolumn{2}{|c|}{$\begin{array}{l}\sim(1-3) \times 10^{2} \\
\sim 10-10^{2} \\
\sim 10-400\end{array}$} & $\begin{array}{l}\sim(3-4.5) \times 10^{13} \\
\sim(4.5-10) \times 10^{13} \\
\sim 10^{14}-3 \times 10^{17}\end{array}$ & $\begin{array}{l}4.5 \times 10^{13} \\
\gtrsim(4.5-10) \times 10^{13} \\
\sim 10^{14}-3 \times 10^{17}\end{array}$ \\
\hline \multicolumn{2}{|c|}{$\begin{array}{c}\text { Radio structure } \\
\text { VLBA }\end{array}$} & \multicolumn{2}{|c|}{$\begin{array}{l}\text { Projected radius } \\
{[\mathrm{AU}]}\end{array}$} & $\begin{array}{l}\text { Velocity } \\
{\left[\mathrm{cm} \mathrm{s}^{-1}\right]}\end{array}$ & \multicolumn{2}{|c|}{ Variability level } \\
\hline \multicolumn{2}{|c|}{ radio core } & $\begin{array}{l}4 \\
\sim 8\end{array}$ & & $\begin{array}{l}\lessgtr 10^{8} \\
\gtrsim 3 \times\end{array}$ & $\begin{array}{l}\approx 10 \% \\
\approx 44 \%(\mathrm{SE}) /\end{array}$ & $60 \%(\mathrm{NW})$ \\
\hline
\end{tabular}

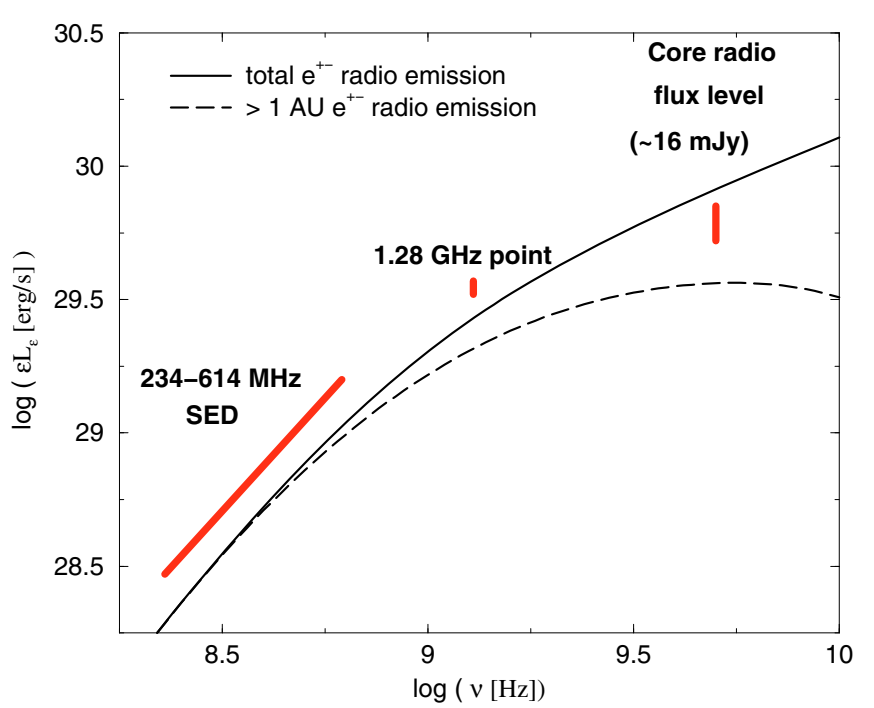

Fig. 2. Computed radio SEDs of the secondary synchrotron emission created by gamma-ray absorption in LS 5039. The SEDs corresponding to the total emission, and to that produced at distances $\geq 1$ AU from the star, are shown. The total differential flux at $5 \mathrm{GHz}$ would be $\approx 20 \mathrm{mJy}$ and, $\geq 1 \mathrm{AU}, \approx 10 \mathrm{mJy}$. The observed luminosities from the $5 \mathrm{GHz}$ core, and at $234 \mathrm{MHz}$ (low state), $614 \mathrm{MHz}$ and $1.28 \mathrm{GHz}$, are also shown (PA0; R08; G08).

inferred in Sect. 2, and also compatible with those in the wind at a few AU from a massive star (Benaglia \& Romero 2003).

Concerning the SE and NW components, the larger $v_{\text {adv }}$ would point to a different physical structure than that producing the radio core, perhaps a real jet. Small changes in the orientation of $\approx 12^{\circ}$ of this jet-like structure have been found (R08). This might be explained, for instance, by an asymmetry introduced by the secondary pairs transported in the stellar wind (B08), the propagation of which might not be spherical, or by jet-stellar wind interactions (Perucho \& Bosch-Ramon 2008).

\subsection{The jet-like structure and the $234 \mathrm{MHz}$ variability}

About a $30 \%$ of the radiation at $5 \mathrm{GHz}$ comes from a jet-like structure of projected size $~ 10-1000$ AU detected with VLBA, MERLIN and EVN (PA0; Paredes et al. 2002; R08). These extended structures may be associated with the $234 \mathrm{MHz}$ highstate emission, due to the size constraints for this region inferred above.

The variability seen at $234 \mathrm{MHz}$ may be related to changes in the outflow properties (e.g. via hydrodynamical instabilities), but not to long-term variations in the primary power supply, since it should affect the core radio emission as well. In this framework, the $234 \mathrm{MHz}$ high state should have associated flux variations at higher frequencies visible outside the radio core. Given that $t_{\mathrm{adv}}$ depends on the size and $v_{\mathrm{adv}}$, the MERLIN and EVN 100-1000 AU structures at $5 \mathrm{GHz}$ would show variability on timescales of at least months. This $234 \mathrm{MHz} /$ large scale jet link can be tested with simultaneous radio VLBI observations at low and high frequencies taken in two different epochs. We note that a radio emitter of $r \sim 1000$ AU varying with $\sim 1 \mathrm{yr}$ timescales would have associated a $v_{\text {adv }} \gtrsim 10^{9} \mathrm{~cm} \mathrm{~s}^{-1}$, compatible with the $v_{\text {adv }}$ derived for the VLBA SE and NW components.

\section{Discussion}

A plausible scenario for the radio emission in LS 5039 would be radiation from secondary pairs created via gamma-ray absorption (see B08), for the compact emitter or core ( $R \sim$ few $\mathrm{AU})$, plus a collimated jet, for the resolved emission.

The jet radio emission from the smaller spatial scales probably would be flat, as typically expected in low-hard state microquasars (e.g. Fender 2001), but the optically thin radiation from secondary pairs would overcome this component, at least up to a few AU. This would explain why the radio spectrum of LS 5039 does not look flat (M98). As observed, this compact radio emission would not present significant changes along the orbit, provided first that the system is not particularly eccentric and, second, that secondary pairs emit radio emission in a region large enough ( $\sim$ few $\mathrm{AU})$ to smooth out any possible modulation of the primary gamma-ray injection. On the other hand, the VLBA, MERLIN and EVN extended radio emission would come from collimated and steady jets. In short, most of the $\gtrsim 614 \mathrm{MHz}$ and the low-state $234 \mathrm{MHz}$ emission would come from the secondary pair radiation, forming the radio core, whereas the high-state $234 \mathrm{MHz}$ emission, and $30 \%$ of the whole $5 \mathrm{GHz}$ radiation, would come from the extended jets.

The 10-1000 AU collimated jets would be consistent with a microquasar nature for LS 5039, and hard to reconcile with the pulsar cometary-tail model (Dubus 2006; Dhawan et al. 2006), although further theoretical work is required before discarding the pulsar scenario, given the complexities inherent to hydrodynamical and magnetohydrodynamical flows (for recent simulations on pulsar winds, see, e.g., Romero et al. 2007; Bogovalov et al. 2008).

The scenario proposed here can be tested looking for: variability in the extended emission at scales $\sim 10 \mathrm{AU}$ (during which the radio core should remain quite steady); further evidence of persistent radio jets beyond the core at scales $\sim 10-1000 \mathrm{AU}$; hints of a spiral-like radio structure (B08) in the still marginally resolved radio core; some kind of correlation between the jetlike radio emission at distances $<100 \mathrm{AU}$ and the $\mathrm{TeV}$ radiation (which varies periodically along the orbit; Aharonian et al. 2006). 
Acknowledgements. We thank Josep Martí for useful suggestions. We thank also an anonymous referee for constructive comments. V.B.-R. gratefully acknowledges support from the Alexander von Humboldt Foundation. V.B.-R. acknowledges support by DGI of MEC under grant AYA2007-68034-C03-01, as well as partial support by the European Regional Development Fund (ERDF/FEDER).

\section{References}

Aharonian, F., Akhperjanian, A. G., Aye, K. M., et al. 2005, Science, 309, 746 Aharonian, F., Akhperjanian, A. G., Bazer-Bachi, A. R., et al. 2006, A\&A, 460, 743

Benaglia, P., \& Romero, G. E. 2003, A\&A, 399, 1121

Bogovalov, S. V., Khangulyan, D., Koldoba, A. V., Ustyugova, G. V., \& Aharonian, F. 2008, MNRAS, 387, 63

Bosch-Ramon, V., Motch, C., Ribó, M., et al. 2007, A\&A, 473, 545

Bosch-Ramon, V., Khangulyan, D., \& Aharonian, F. 2008a, A\&A, 482, 397 (B08)

Bosch-Ramon, V., Khangulyan, D., \& Aharonian, F. 2008b, A\&A, 489, L21

Casares, J., Ribó, M., Ribas, I., et al. 2005, MNRAS, 364, 899 (C05)

Dhawan, V., Mioduszewski, A., \& Rupen, M. 2006, The VI Microquasar Workshop: Microquasars and Beyond, Proc. Science, 52, 1
Dubus, G. 2006, A\&A, 456, 801

Fender, R. 2001, MNRAS, 322, 31

Godambe, S., Bhattacharya, S., Bhatt, N., \& Manojendu, C. 2008, MNRAS, 390, 43 (G08)

Khangulyan, D., Aharonian, F., \& Bosch-Ramon, V. 2008, MNRAS, 383, 467 Krtidka, J., \& Kubát, J. 2001, A\&A, 377, 175

Martí, J., Paredes, J. M., \& Ribó, M. 1998, A\&A, 338, L71 (M98)

Martocchia, A., Motch, C., \& Negueruela, I. 2005, A\&A, 430, 245

Owocki, S., \& Cohen, D. 2006, ApJ, 648, 565

Pacholczyk, A. G. 1970, Radio Astrophysics (San Francisco, CA: Freeman) Pandey, M., Rao, A. P., Ishwara-Chandra, C. H., Durouchoux, P., \& Manchanda, R. K. 2007, A\&A, 463, 567 (P07)

Paredes, J. M., Martí, J., Ribó, M., \& Massi, M. 2000, Science, 288, 2340

Paredes, J. M., Ribó, M., Ros, E., Martí, J., \& Massi, M. 2002, A\&A, 393, L99

Paredes, J. M., Bosch-Ramon, V., \& Romero, G. E. 2006, A\&A, 451, 259

Perucho, M., \& Bosch-Ramon, V. 2008, A\&A, 482, 917

Ribó, M., Paredes, J. M., Moldón, J., Martí, J., \& Massi, M. 2008, A\&A, 481, 17 (R08)

Romero, G. E., Okazaki, A. T., Orellana, M., \& Owocki, S. P. 2007, A\&A, 474, 15

Rybicki, G. B., \& Lightman, A. P. 1979, Radiative processes in astrophysics (New York: Wiley-Interscience) 